\title{
Kinetics of Isothermal Annealing of Gamma-irradiation Damage in Thallous Nitrate
}

\author{
JADU SAMUEL* and SUNIL CULAS \\ Department of Chemistry, Research Centre, Mar Ivanios College, \\ Thiruvananthapuram - 695 015, Kerala, India. \\ ${ }^{*}$ Corresponding author E-mail: jadu_samuel@yahoomail.com \\ http://dx.doi.org/10.13005/ojc/290433
}

(Received: September 16, 2013; Accepted: November 08, 2013)

\begin{abstract}
Thallous nitrate, $\mathrm{TINO}_{3}$ was irradiated with ${ }^{60} \mathrm{Co} \gamma$-rays to $7 \times 10^{2} \mathrm{KGy}$ at a dose rate of $2 \mathrm{kGy} \mathrm{h}^{-1}$. The FT-IR spectrum was used to confirm the existence of damage nitrite while crystal transformations in $\mathrm{TINO}_{3}$ were established by TG/DTA analysis. The annealing data in the temperature range, 393-433 $\mathrm{K}$ was analysed using Fletcher-Brown, Waite and Conventional chemical kinetic methods in order to determine the order of the recovery process, the energy of activation and the fraction recovered as a function of temperature. Thermal annealing is found to be a combination of a fast first order process affecting a greater proportion of the fragments, followed by a predominantly slow second order process, with a higher energy of activation, governing the behaviour of the remainder. It is considered that the first order process is the combination of close-correlated pairs of $\mathrm{O}$ and $\mathrm{NO}_{2}$ fragments and the second order process involves the single reaction of random recombination of the fragments throughout the crystal.
\end{abstract}

Key words: Thallous nitrate, Gamma rays, Isothermal annealing, Irradiation damage, Activation energy.

\section{INTRODUCTION}

The effects produced in solids by bombardment depend on nature of the target and energy of the incident radiation. The important effects are excitation, ionisation, atomic displacement from lattice sites and transmutation of nuclei which are either primary or secondary depending on the sequence of the events following the absorption of energy ${ }^{1-3}$. The primary effect of neutron irradiation is atomic displacement with ionisation as a secondary process. On the other hand, irradiation with $g$-rays produces ionisation as the important primary effect, atomic displacements sometimes results secondarily ${ }^{4-6}$.

The chemical damage induced in systems containing oxyanions, such as nitrates, bromates, chlorates and perchlorates has been reviewed by Zakharov and Novostruev ${ }^{7}$ and by Johnson ${ }^{8}$. The stability of radiolytic product varies widely with crystal matrix ${ }^{9}$. It was observed by Maddock and Mohanty ${ }^{10}$ that macroscopic chemical damage induced by energetic radiations is susceptible to recovery (annealing) in the same way as microscopic chemical effects of nuclear transformations and 
physical effects of irradiation. They observed that when radiolysed solid metal nitrates are heated the damage nitrite diminishes progressively with time. The disappearance of the nitrite was due to the recombination of the damage fragments which remain trapped in the lattice to form the original nitrate. Following this, several research groups ${ }^{11-18}$ have studied the radiolysis and recovery of damage fragments in irradiated nitrates of alkali and alkaline earth metals as well as complex compounds. The extent of annealing may differ from system to system depending on the physical and chemical properties of the host crystals and upon that of the irradiation employed ${ }^{9}$.

In the case of irradiated metallic nitrates which show crystal transformations on heating, the recovery processes are taking place due to thermal reactions as well as reactions accompanying phase transformations in the damaged crystals ${ }^{19}$. It was reported ${ }^{20}$ that the yield of both the nitrite and oxygen formed by the radiolysis of potassium nitrate was reduced by post irradiation heating of the nitrate above the phase transition temperature (rhombic'!trigonal at $402 \mathrm{~K}$ ). It was observed by Clark and Reinhardt ${ }^{21}$ that $\mathrm{TINO}_{3}$ shows two crystal transformations on heating. When heated to about $350 \mathrm{~K}$ the orthorhombic crystals transform into a rhombohedral form, which in turn changes to cubic at $418 \mathrm{~K}$. So it is of interest to investigate the effect of $g$-irradiation and subsequent isothermal annealing of chemical radiation damage in $\mathrm{TINO}_{3}$ at different temperatures in the range $393-433 \mathrm{~K}$. This has been carried out with a view to determine the order of the recovery process and the energy of activation on the basis of diffusion controlled mechanisms ${ }^{22,23}$ as well as Conventional chemical kinetic methods ${ }^{24}$.

\section{EXPERIMENTAL}

\section{Irradiation}

Analytical reagent grade $\mathrm{TINO}_{3}$ (SigmaAldrich) was used. The sample was sieved to obtain crystals in the 85-100 mesh range. Samples of $\mathrm{TINO}_{3}$ sealed in glass ampoules were irradiated at room temperature with ${ }^{60} \mathrm{Co} \gamma$-rays to a dose of $7 \times 10^{2} \mathrm{KGy}$ at a dose rate of $2 \mathrm{KGy} \mathrm{h}^{-1}$. The irradiated samples were thoroughly mixed and stored over phosphorus pentoxide.

\section{Analytical procedure}

The damage nitrite was estimated spectrophotometrically by the method developed by Shinn ${ }^{25}$ and modified by Kershaw and Chamberlin ${ }^{26}$. The method was shown to be sensitive to $3 \mathrm{ppm}$ of nitrite. The colour was found permanent up to $70 \mathrm{~min}$ after development.

In the routine measurements, $0.2-0.3 \mathrm{~g}$ of the irradiated nitrate crystals were dissolved in boiled out distilled water to give $100 \mathrm{~mL}$ of solution. A suitable aliquot of this solution was transferred to a $50 \mathrm{~mL}$ standard flask and $2 \mathrm{~mL}$ of a $0.5 \%$ solution of sulphanilamide in 1:1 hydrochloric acid added. After $3 \mathrm{~min}, 1 \mathrm{~mL}$ of a $0.1 \%$ solution of $\mathrm{N}$-(1-naphthyl) ethylenediamine dihydrochloride was added. After mixing, the solution was made up to $50 \mathrm{~mL}$ and allowed to stand $15 \mathrm{~min}$ before measurement of the absorbance at $540 \mathrm{~nm}$.

\section{FT-IR spectrum}

The FT-IR spectrum of both untreated and irradiated $\mathrm{TINO}_{3}$ was recorded on $\mathrm{KBr}$ pellets using Schimadzu spectrophotometer.

\section{TG/DTA analysis}

Thermogravimetric (TG) and differential thermal analysis (DTA) of untreated $\mathrm{TINO}_{3}$ was performed on an SII NanoTechnology TG/DTA 6200 instrument with pure nitrogen $\left(50 \mathrm{~mL} \mathrm{~min}^{-1}\right)$ as a carrier gas at a heating rate of $5^{\circ} \mathrm{C} \mathrm{min}$ msing $^{-1}$ using aluminium crucible.

\section{Thermal annealing}

Isothermal annealing runs were made with the irradiated crystals in air for different time intervals up to $20 \mathrm{~h}$ at various temperatures in the range 393$433 \mathrm{~K}$ and also at a temperature $343 \mathrm{~K}$ (below phase transition temperature) in a thermostatic electric hotair oven maintained to within $\pm 1^{\circ} \mathrm{C}$ of the desired temperature.

\section{RESULTS AND DISCUSSION}

\section{IR spectral studies}

FT-IR spectra of untreated and irradiated $\mathrm{TINO}_{3}$ are depicted in Figure.2. An intense and wide peak centered at $\sim 1364 \mathrm{~cm}^{-1}$ (Figure 2a) is due to asymmetric stretching vibrations of $\mathrm{NO}_{3}^{-}$ion. 
Table 1: Temperature variation of the first and second order velocity constants in the annealing of $\gamma$-irradiated $\mathrm{TINO}_{3}$

\begin{tabular}{lll}
\hline $\begin{array}{l}\text { Temperature (K) } \\
\left.\mathbf{h}^{-1}\right)\end{array}$ & $\boldsymbol{k}_{\text {I }}\left(\mathbf{h}^{-1}\right)$ & $\boldsymbol{k}_{\text {II }} \times \mathbf{1 0}^{4}\left(\mathbf{p p m}^{-1}\right.$ \\
393 & & \\
403 & 0.6266 & 0.3936 \\
413 & 0.7623 & 0.5404 \\
423 & 0.8756 & 0.8668 \\
& 1.0665 & 1.4918 \\
\hline
\end{tabular}

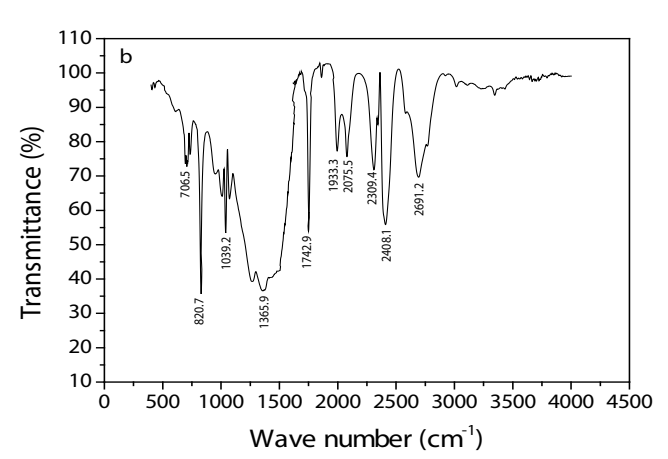

Fig. 1: FT-IR spectra of TINO $_{3}$ : a, unirradiated and b, $\gamma$-irradiated

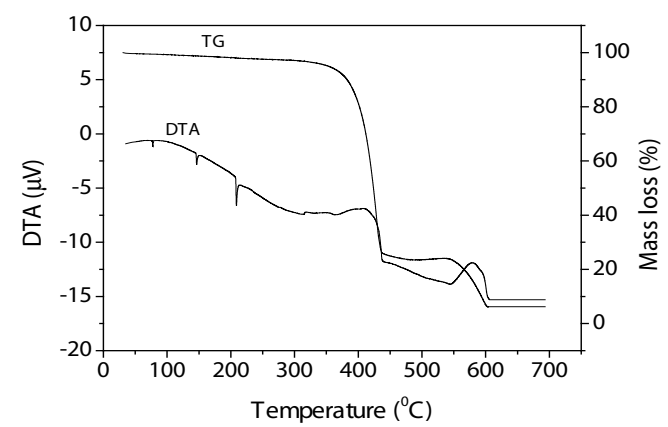

Fig. 2: TG and DTA curves of untreated $\mathrm{TINO}_{3}$

$1380-1350$ and $840-815 \mathrm{~cm}^{-1}$. In another article ${ }^{31}$ it is reported that asymmetric and symmetric stretching of Free State of $\mathrm{NO}_{3}^{-}$occurs at $1355,1049 \mathrm{~cm}^{-1}$ respectively whereas the asymmetric and symmetric bending of $\mathrm{NO}_{3}^{-}$appears around at $830,690 \mathrm{~cm}^{-1}$ respectively. A comparison of FT-IR spectra of unirradiated and irradiated $\mathrm{TINO}_{3}$ shows that spectral region of $1500-1000 \mathrm{~cm}^{-1}$ for irradiated samples have some changes in frequency and intensity of vibrational bands. This difference essentially arises due to the interactions between the vibrations of $\mathrm{NO}_{3}^{-}$and $\mathrm{NO}_{2}{ }^{-}$ions in the lattice. This clearly provides
The peak at $1044 \mathrm{~cm}^{-1}$ is attributed to symmetric stretching vibrations of $\mathrm{NO}_{3}^{-}$ion. The asymmetric and symmetric bending of $\mathrm{NO}_{3}{ }^{-}$appears at 820 and $711 \mathrm{~cm}^{-1}$ respectively.

It has been already reported that inorganic nitrate and nitrite groups show four and three fundamental modes of vibrations, respectively, in IR region ${ }^{27,30}$. Nitrate has four modes of vibrations at $1370,1020,840$ and $730 \mathrm{~cm}^{-1}$, but only two characteristic bands are observed in the vicinity of
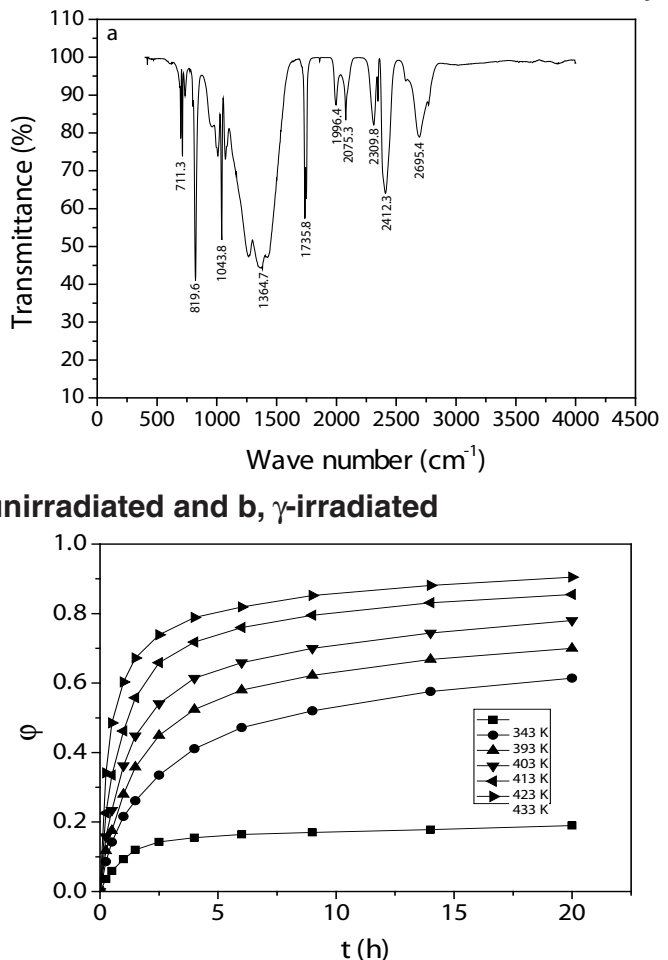

Fig. 3: Plot of fraction annealed $(j)$ against heating time $(t)$ for the isothermal annealing of $\gamma$ - irradiation damage in $\mathrm{TINO}_{3}$

additional evidence besides spectrophotometric estimation, for the formation of $\mathrm{NO}_{2}^{-}$ions formed upon ã-irradiation on $\mathrm{TINO}_{3}$. The observed change in pattern of FT-IR spectrum in the spectral region $1000-1500 \mathrm{~cm}^{-1}$ for untreated and irradiated $\mathrm{TINO}_{3}$ in the present work (Figure 2) is similar to that reported for $\gamma$-irradiated $\mathrm{Ba}\left(\mathrm{NO}_{3}\right)_{2}^{32}$.

\section{TG/DTA curves}

Figure 2 shows TG/DTA curves for untreated $\mathrm{TINO}_{3}$. Two solid-solid transition points and the melting point observed from DTA curve are 


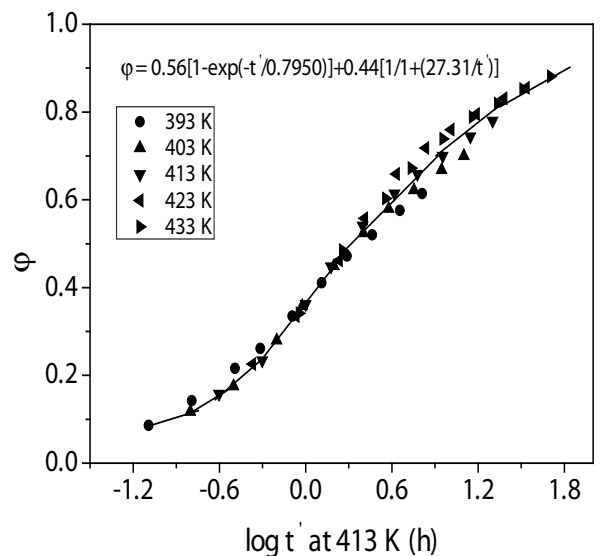

Fig. 4: The Fletcher-Brown composite annealing curve of fraction annealed (j) against logarithm

of equivalent time $\left(\log t^{\prime}\right)$ at a reference temperature of $413 \mathrm{~K}$ for the isothermal annealing of $\gamma$-irradiation damage in $\mathrm{TINO}_{3}$

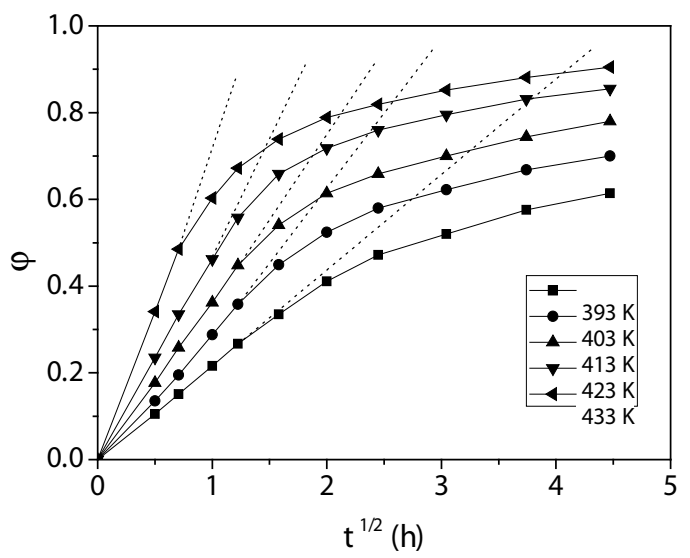

Fig. 6: Annealing as a function of the square root of heating time $\left(t^{1 / 2}\right)$ for $\gamma$-irradiated TINO $_{3}$

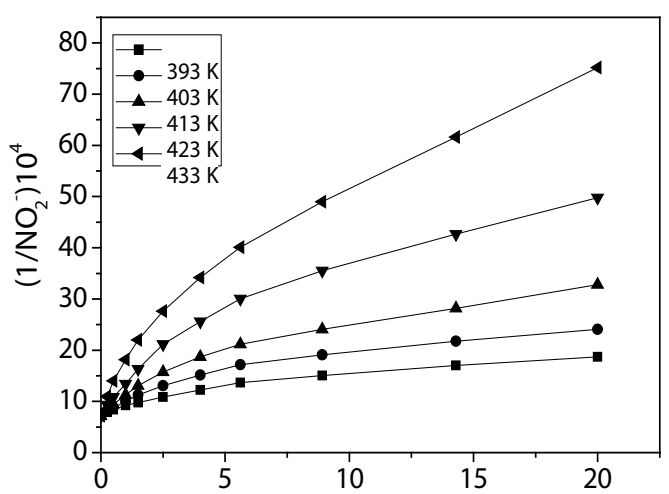

Fig. 8: The second Heating time (hr) of $\gamma$-irradiation damage in $\mathrm{TINO}_{3}$

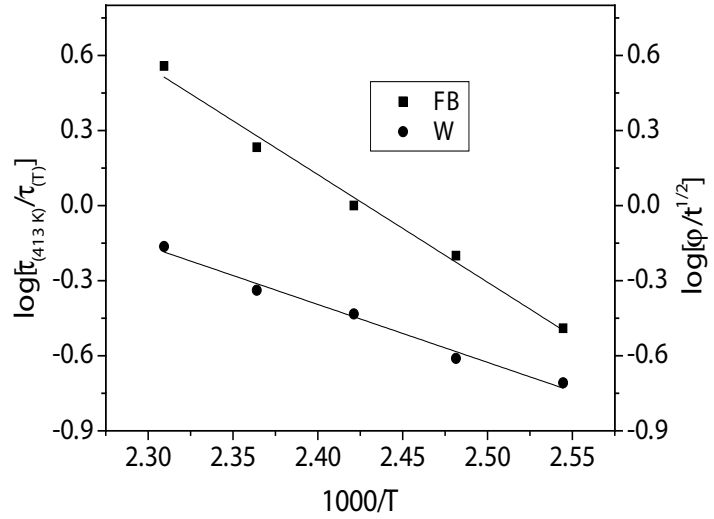

Fig. 5: Energetics of Fletcher-Brown (FB) and Waite (W) model for $\gamma$-irradiated $\mathrm{TINO}_{3}$ in the temperature range $393-433 \mathrm{~K}$

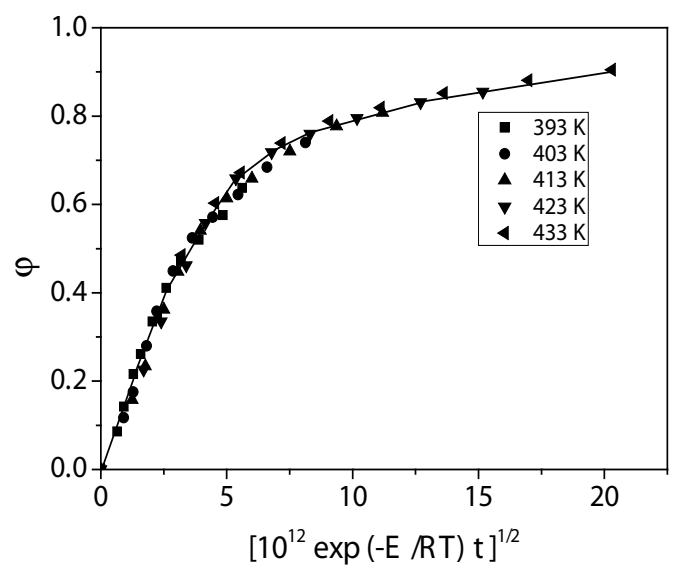

Fig. 7: The Waite composite annealing curve for $\gamma$-irradiated $\mathrm{TINO}_{3}$

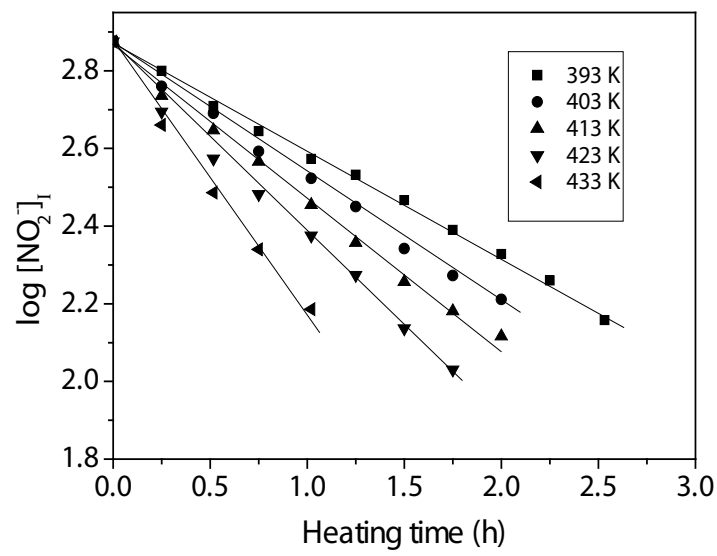

Fig. 9: The first-order contribution in annealing of $\gamma$-irradiation damage in $\mathrm{TINO}_{3}$ 


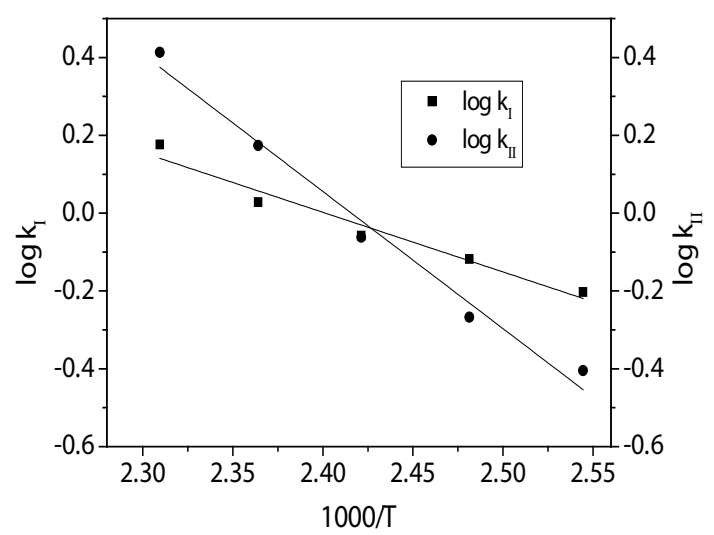

Fig. 10: Energetics of conventional chemical kinetics for $\gamma$-irradiated TINO $_{3}$ in the temperature range $393-433 \mathrm{~K}$

$350 \mathrm{~K}, 420 \mathrm{~K}$ and $481 \mathrm{~K}$, respectively. TG showed a weight loss beginning at $\sim 663 \mathrm{~K}$ with a 50 percent loss by $\sim 793 \mathrm{~K}$. This weight loss was demonstrated to be caused by boiling rather than decomposition. The DTA extrapolated onset temperature for the boiling was $719 \mathrm{~K}$. The similar observations have been reported earlier for the TG/DTA study of $\mathrm{TINO}_{3}$ by Clark and Reinhardt ${ }^{21}$.

\section{Isothermal annealing curves}

The irradiation produced a golden yellow coloration in the crystals. The initial damage produced by $7 \times 10^{2} \mathrm{KGy}$ of ${ }^{60} \mathrm{Co}$ g-rays in $\mathrm{TINO}_{3}$ was $1386 \mathrm{ppm}$ of nitrite. The bleaching of the colour produced and the recovery of damage upon heating of the irradiated crystals were similar to those reported earlier ${ }^{24,18}$.

The fraction annealed $j$ in terms of nitrite concentration on heating the irradiated sample at a given temperature is given by:

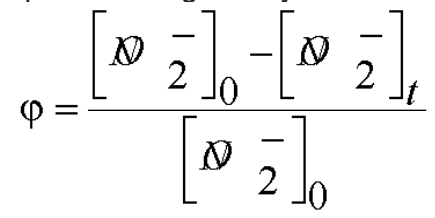

where $\left[\mathrm{NO}_{2}^{-}\right]_{0}$ and $\left[\mathrm{NO}_{2}^{-}\right]_{t}$ are the values of nitrite concentration at initial and after a time $t$ respectively at a given temperature. The annealing pattern for damaged nitrite in $\mathrm{TINO}_{3}$ at various temperatures in the range $393-433 \mathrm{~K}$ and also at $343 \mathrm{~K}$ is presented in Figure 3 . The recovery of damage below phase transition temperature is very slow as shown by the curve at $343 \mathrm{~K}$. But the fraction annealed $j$, in the range $393-433 \mathrm{~K}$ first increases rapidly and then slowly with heating time, $t$. This abrupt recovery of damage is due to thermal reactions as well as reactions accompanying phase transformations as reported earlier ${ }^{33,34,19}$ in the damaged crystals.

\section{Kinetics of isothermal annealing}

In order to determine the order and the energy of activation for the recovery process of $g$-irradiation damage in $\mathrm{TINO}_{3}$, the annealing data has been analysed using three different kinetic methods: Flethcher and Brown, Waite and Conventional chemical kinetic methods. The details of these methods have already been reported ${ }^{11,16}$ 17.

\section{The Fletcher-Brown model}

Fletcher and Brown 22 and Brown et al. ${ }^{35}$ have considered the theoretical aspects of recombination. According to Fletcher and Brown, the recombination occurs in three stages, viz., close pair, liberation and second order, and it is governed by a single energy of activation so that recovery is a unique function of equivalent time, $t$.

$$
t^{\prime}=\frac{t}{\tau}=t\left[D_{0} \exp \left(\frac{-E}{\mathbb{E}}\right)\right]=D
$$

where $\tau=1 / D=D_{0}^{-1} \exp (E / k T)$, the diffusive jump time in the lattice, $t$ the heating time, $t$ the equivalent time, $D_{0}$ the constant frequency term, $E$ the energy of activation formation, $k$ the Boltzmann constant, $T$ the heating temperature, and $D$ the diffusion coefficient. Since the annealing is a function of t/ô only, it is possible that data at different temperatures and time intervals can be adequately superimposed on to a single universal curve by adjusting the time scales by proper factors. The application of Fletcher and Brown model to our data for the annealing reactions in $g$-irradiated $\mathrm{TINO}_{3}$ is shown in Figure 4 in which the data are plotted against logarithm of equivalent time at a reference temperature of $413 \mathrm{~K}$. The data are adequately superimposed and are represented by the continuous curve which is the theoretical fit of the following combination of a fast first order and a slow predominant second order process: 
$\varphi=0.5\left[1-\exp \left(\frac{-t^{\prime}}{0.79502}\right)\right]+0.4\left[\frac{1}{1+\left(2.3088 / t^{\prime}\right)}\right]$

The logarithm of the relative jump time $\tau_{(413}$ k) $/ \tau_{(T)}$ is linearly related to $1 / T$ (Figure 5 ), and the activation energy computed is $19.7 \mathrm{k} \mathrm{cal} \mathrm{mol}^{-1}$.

\section{The Waite model}

Waite has treated the entire phenomenon of recovery as diffusion limited reaction. The resulting expression for the kinetics of recovery is very complex, but for small initial defect concentrations and for early times:

$$
\varphi=K(D)^{1 / 2}
$$

where $j$ is the fraction of the damage annealed at time $t, D$ the diffusion coefficient and $K$ a quantity independent of temperature. The change of $j / t^{1 / 2}$ for early times with temperature gives the temperature dependence of $D$, which is of the form:

$$
D=D_{0} \exp \left(\frac{-E}{\mathscr{H}}\right)
$$

In this case, the recombination is governed by a single energy of activation, as in the case of Fletcher-Brown model. The Waite plots for annealing of irradiation damage in $\mathrm{TINO}_{3}$ at different temperatures are shown in Figure 6, as a graph of $j$ against $t^{1 / 2}$. It is seen that the curves are linear for early times in agreement with the Waite theory. The activation energy for diffusion is calculated from the temperature dependence of $j / t^{1 / 2}$ as $21.2 \mathrm{k}$ cal $\mathrm{mol}^{-1}$ (Figure 5), and this value is comparable to that obtained in the Fletcher-Brown treatment of the data. Figure 7 shows that the annealing data taken at different temperatures can be represented by single universal curve, by a proper adjustment of the time factor, as a unique function of $\left[D_{0} \exp (-E / R T) t\right]^{1 / 2}$ so that the data are adequately superimposed in this universal curve. The initial linearity of this curve is also evident from Figure 7, in agreement with the Waite theory.

\section{Analysis on the basis of conventional chemical kinetics}

The annealing data have also been considered in the light of Conventional chemical kinetics and is found that plots of the reciprocal of the nitrite concentrations are a function of time (Figure 8). The linear portions of the curves do not extrapolate to the same intercept on the $t=0$ axis because with rise in temperature, lattice mobility increases, liberating more and more atomic oxygen facilitating the recombination process (fast phase change annealing). The slopes of linear portions of these curves (Figure 8) give values of second order velocity constants, $k_{\| \mid}$at each annealing temperature. Except for an initial period of the recovery, linearity is obtained in all cases suggesting a bimolecular reaction. The first order contribution for different species are obtained by extrapolating the second order plots at various temperatures to zero time and the difference between read off and experimental values at a particular time are taken. Plots of logarithm of these values against heating time are linear (Figure 9) whose slope will give first order velocity constants, $k_{1}$ at each annealing temperature. The values of $k_{1}$ and $k_{\|}$are given in Table 1. The Arrhenius plots of log $k_{1}$ and $\log$ $k_{\text {II }}$ against $1 / T$ (Figure 10) yield the activation energy 29.33 and $79.77 \mathrm{~kJ} \mathrm{~mol}^{-1}$ for the first and second order process respectively. Thus, as is evident from the Fletcher-Brown treatment of the data, annealing can be represented by a combination of a unimolecular and a bimolecular process. The mean values of the proportions of the original nitrite, which recombine by the first and second order mechanisms, are $56 \%$ and $44 \%$, respectively.

\section{Chemistry of annealing}

The existence of a number of species in irradiated nitrate has been reported ${ }^{36,14,17}$. With moderate doses of irradiation at room temperature, the damage fragments are mainly $\mathrm{NO}_{2}, \mathrm{O}$ and $\mathrm{O}_{2}$ with low concentration of $\mathrm{NO}, \mathrm{NO}_{2}, \mathrm{O}_{2}^{-}, \mathrm{O}_{3}^{-}$etc. The charged fragment is situated in the lattice position of the original ion and the neutral fragments some distance away from the damage site. In the annealing process, the neutral fragments undergo diffusive movement and reacts with the charged fragments to reform the target ion ${ }^{19}$.

The fast phase change recovery may be due to the rapid reaction of $\mathrm{NO}_{2}^{-}$and $\mathrm{O}$ pairs as follows: 


$$
\mathrm{NO}_{2}^{-}+\mathrm{O} \rightarrow \mathrm{NO}_{3}^{-}
$$

The first order disappearance of the nitrite is the recovery of closely correlated $\mathrm{NO}_{2}^{-}$and $\mathrm{O}$ pairs:

$$
\mathrm{NO}_{2}^{-}+\mathrm{O} \rightarrow \mathrm{NO}_{3}^{-}
$$

The second order reaction is the random recombination of $\mathrm{NO}_{2}^{-}$and $\mathrm{O}$ or $\mathrm{O}_{2}$ fragments which have diffused farther apart. The second order reaction of nitrite and atomic oxygen is only possible when the species are present in equal concentration but there will be competition between the following reactions:

and

$$
\mathrm{O}+\mathrm{O} \rightarrow \mathrm{O}_{2}
$$

$$
\mathrm{NO}_{3}^{-}+\mathrm{O} \rightarrow \mathrm{NO}_{2}^{-}+\mathrm{O}_{2}
$$

with reaction (7). The trapped oxygen released upon dissolution is shown to be primarily molecular in nature ${ }^{8}$. The more plausible second order process is:

$$
2 \mathrm{NO}_{2}^{-}+\mathrm{O}_{2} \rightarrow 2 \mathrm{NO}_{3}^{-}
$$

\section{CONCLUSIONS}

The FT-IR and spectrophotometric estimations provide additional evidence for the formation of $\mathrm{NO}_{2}^{-}$ions upon $\tau$-irradiation of $\mathrm{TINO}_{3}$. DTA has been used to confirm previously reported temperatures for two solid-solid transformations and for the melting of $\mathrm{TINO}_{3}$. The present investigations revealed that the recovery of damage in irradiated sample occurs due to thermal reactions as well as reactions accompanying phase transformations in the damaged crystals. The isothermal annealing process is found to consist of a fast first-order reaction, in addition to a slow predominant second-order reaction. It has been suggested that the unimolecular process corresponds to the recombination of closely correlated $\mathrm{NO}$ and $\mathrm{O}_{2}^{-}$pairs and the second order process to the random recombination of the damage fragments throughout the crystal.

\section{ACKNOWLEDGEMENTS}

The authors gratefully thank the Rubber Board, Kottayam, Kerala for lending irradiation facility.

\section{REFERENCES}

1. N. F. Mott and H. S. W. Massey, The Theory of Atomic Collisions, Clarendon Press, Oxford, 1949.

2. H. S. W. Massey and E. H. S. Burhop, Electronic and Ionic Impact Phenomena, Clarendon Press, Oxford, 1952.

3. F. Seitz and J. S. Koehler, Displacement of Atoms During Irradiations: Solid State Physics (Ed. F. Seitz, D. Turnbull) vol. 2, Academic Press, New York, 1956.

4. E. P. Wigner, J. Appl. Phys., 17: 857 (1946).

5. M. Burton, J. Phys. Colloid. Chem., 51: 611 (1947).

6. F. Seitz, Discuss. Faraday. Soc., 5: 271 (1949).

7. Y. A. Zakharov and V. A. Nevostruev, Russ. Chem. Rev., 37: 61 (1968).

8. E. R. Johnson, The Radiation Induced Decomposition of Inorganic Molecular Ions,
Gordon and Breach Science Publishers, New York, 1970.

9. R. M. Golding and M. Henchman, J. Chem. Phys., 40:1554 (1964).

10. A. G. Maddock and S. R. Mohanty, Nature, 182: 1797 (1958).

11. A. G. Maddock and S. R. Mohanty, Discuss. Faraday. Soc., 31: 193 (1961).

12. S. Kaucic and A.G. Maddock, Trans. Faraday. Soc., 65: 1083 (1969).

13. S. R. Mohanty and P. Patra, Radiochim. Acta, 23: 152 (1976).

14. D. Muhammad and A. G Maddock, J. Chem. Soc. Faraday. Trans., 174: 919 (1978).

15. D. Bhatta, S. R. Mohanty and K.C. Samantaray, Radiochim. Acta, 28: 13 (1981).

16. D. Bhatta, P. Patra, and S. R. Mohanty, Indian. J. Chem. A, 21: 805 (1982).

17. D. Bhatta, P. Patra, and S. R. Mohanty, 
Radiochim. Acta, 35: 205 (1984).

18. S. M. K. Nair and C. James, Radiat. Eff., 62: 211 (1982).

19. B. M. Mohapatra and D. Bhatta, Radiat. Phys. Chem., 27: 339 (1986).

20. Proskurnin, Orekhov, Barelko Conf. Acad. Sci. U.S.S.R. on Peaceful Uses of Atomic Energy (Chem. Sci. Div.) p. 41(1955).

21. R.P. Clark and F.W. Reinhardt, J. Therm. Anal., 13: 321(1978).

22. R. C. Flethcher and W. L. Brown, Phys. Rev., 92: 585 (1953).

23. T. R. Waite, Phys. Rev., 107: 463 (1957).

24. S. R. Mohanty and S. R. Upadhyay, Z. Phys. Chem. Neue., 57: 234 (1968).

25. M. B. Shinn, Ind. Engng. Chem., 13: 33 (1941).

26. N. F Kershaw and N. S. Chamberlin, Ind. Engng. Chem.,14: 312 (1942).

27. C. N. R. Rao, Chemical Applications of Infrared
Spectroscopy, Academic Press, New York, 1963.

28. K. Nakamoto, Infrared and Raman Spectra of Inorganic and Coordination Compounds, third ed. Wiley, New York (1978).

29. Om Prakash and G. Jacob, Orient J. Chem., 29(3): 1079-1084 (2013).

30. Neeti Rathore and B. Krishan, Orient J. Chem., 29(3): 1001-1008 (2013).

31. J. Emsley, Chem. Soc. Rev., 9: 91 (1980).

32. N. Agrawal and A. N. Garg, Radiat. Phys. Chem., 73: 147 (2005).

33. B. M. Mohapatra and D. Bhatta, Indian. J. Chem. A, 23: 512 (1984).

34. V. Chakravortty, J. K. Mishra and S. R. Mohanty, Indian. J. Chem., 20: 77 (1981).

35. W. L. Brown, R. C. Flethcher and K. A. wright, Phys. Rev., 92: 591 (1953).

36. J. Cunningham, J. Phys. Chem., 67: 1771 (1963). 